\title{
Acinetobacter baumannii: Identification, Antibiotic Sensitivity and Biofilm Formation in Different Clinical Samples
}

\author{
Maherun Nesa ${ }^{1}$, Shaheda Anwar ${ }^{2}$, Ahmed Abu Saleh ${ }^{2}$ \\ ${ }^{1}$ Department of Microbiology, Dhaka Medical Collage, ${ }^{2}$ Department of Microbiology and immunology, Bangabandhu Sheikh Mujib \\ Medical University
}

\begin{abstract}
Background: Acinetobacter baumannii is responsible for nosocomial infections which are related to biofilm formation of this pathogen. Biofilm formation helps the bacteria in surviving stressed environmental conditions and bacteria growing in biofilms are resistant to most of the commonly used antibiotics.

Objectives: The objective of this study was to detect A. baumannii, to see antibiotic sensitivity and biofilm formation in different clinical samples.

Methods: Total 108 Acinetobacter spp. were collected from different clinical samples which were identified by conventional microbiological procedures. Out of 108 Acinetobacter spp, 85 were identified as A. baumannii by polymerase chain reaction by detecting blaOXA-51 gene which is intrinsic to A. baumannii. Antibiotic sensitivity was detected by modified disc diffusion method and biofilm formation was detected by Tissue culture plate method.

Results: Among 85 isolates, $45.9 \%$ A. baumannii were obtained from tracheal aspirate followed by blood (21.2\%), wound swab (15.3\%), urine (10.6\%), pus (5.9\%) and pleural fluid (1.1\%). More than $80 \%$ Of A. baumannii was resistant to cephalosporin, aminoglycosides, quinolone, carbapenem. By Tissue culture plate method, $78.8 \%$ of isolates showed biofilm formation. Biofilm formation in tracheal aspirate was $82.1 \%$, in blood $72 \%$, in wound swab $92 \%$, in urine $44.4 \%$, in pus $100 \%$ and in pleural fluid $100 \%$.
\end{abstract}

Conclusion: Detection rate of A. baumannii was more in tracheal aspirates. Biofilm producing A. Baumannii was resistant to most of the antibiotics.

Key words: Biofilm, Tissue culture plate method, Polymerase chain reaction

\section{Introduction}

Thirty-three genomic species (gen. sp.) of the Acinetobacter genus have so far been identified of which Acinetobacter baumannii is an important nosocomial pathogen that is responsible for a wide range of human infections such as pneumonia, septicemia, wound sepsis, urinary tract infections, endocarditis and meningitis. ${ }^{1,2}$ A. baumannii, genomic species 3 and 13TU, three of the most clinically relevant species, are genetically and phenotypically very similar to an environmental species, Acinetobacter calcoaceticus, and are therefore grouped together into the so-called A. calcoaceticus-Acinetobacter baumannii complex. ${ }^{1}$ Because antibiotic susceptibility and clinical relevance are significantly different between different genomic

Correspondence:

Dr. Maherun Nesa

Medical Officer

Department of Microbiology

Dhaka Medical College, Shahbagh, Dhaka-1000.

Tel : +8801740336877, Email : munbsmmu@gmail.com species, exact identification of Acinetobacter species are required. ${ }^{2}$ Identification within the genus is difficult and requires molecular methods. ${ }^{3}$ Several genotypic methods have been developed for genomic species identification, which include amplified 16S rRNA gene restriction analysis, highresolution fingerprint analysis by amplified fragment length polymorphism, sequence analysis of the 16S-23S rRNA gene spacer region, $r p o B$ sequencing and $g y r B$ multiplex PCR. ${ }^{4}$ The recA gene ${ }^{5}$ and blaOXA-51-like gene ${ }^{5,6}$ were also used for A. baumannii genotypic identification. The genes blaOXA-51-like is intrinsically present in A. baumannii. ${ }^{7}$

Recently, the rapid development of multiple antibiotic resistance of A. baumannii has caused a serious problem for public health. The ability of biofilm formation contributes to Acinetobacter easily survive and transfer in the hospital environment, such as attached to various biotic and abiotic surfaces, e.g., vascular catheters, cerebrospinal fluid shunts or Foleys catheter. ${ }^{8}$ In the clinical samples, the most commonly 
encountered opportunistic pathogen is A. baumannii and because of its ability for colonization to the hospital setting and developing resistance, it leads to nosocomial infections that are difficult to treat. ${ }^{9}$ Biofilm formation on invasive device by strains of Acinetobacter spp. is considered to be an important virulence factor for such infections. ${ }^{10}$ Biofilm formation not only helps to protect the bacteria, but also helps in trading resistance genes between the participating cells. The ability of some A. baumannii isolates to produce biofilms might also explain its outstanding antibiotic resistance, survival properties and increased its pathogenicity. ${ }^{11}$ This infection are difficult to eradicate as Acinetobacter spp. growing in biofilm are resistant to most of the antimicrobials thereby limiting therapeutic options. Biofilm formation on surfaces and expression of multidrug resistance favours dissemination of Acinetobacter spp. in hospital setting. ${ }^{10}$

\section{Materials and Methods}

This cross sectional study was conducted at the Department of Microbiology \& Immunology, Bangabandhu Sheikh Mujib Medical University (BSMMU), Dhaka, Bangladesh from September 2017 - August 2018.

\section{Bacterial Isolates and Laboratory Identification of Acinetobacter spp.}

Total 108 Acinetobacter spp. were collected from different clinical samples (tracheal aspirate, wound swab, pus, blood, urine, pleural fluid). The clinical samples were sent from different ward of Bangabandhu Sheikh Mujib Medical University Hospital in Department of Microbiology \& Immunology (BSMMU) for culture and antimicrobial sensitivity test. Acinetobacter spp. were identified by culture, Gram stain and biochemical tests (catalase test, oxidase test, urease test, motility test, citrate utilization test and Oxidation-Fermentation test).

\section{Identification of $A$. baumannii by PCR}

From 108 Acinetobacter spp. 85 were confirmed as A. baumannii by identification of the blaOXA-51-like gene. The PCR was performed in culture isolates using specific primers for detection of blaOXA-51-like gene (Applied biosystems, Thermofisher scientific, USA). DNA was extracted from bacterial colonies by boiling method. Two colonies of overnight growth of A. baumannii were taken in a $2 \mathrm{ml}$ micro centrifuge tube (ExtraGene, Taiwan). One $\mathrm{ml}$ of distilled water was added and boiled in a heat block (Incublock, Denville scientific inc. USA) for 10 minutes at $100^{\circ} \mathrm{C}$. The tubes were then centrifuged for 5 minutes at $168 \mathrm{~g}$ (Hermle Z $233 \mathrm{M}-2$, Labnet international inc. USA). Supernatant was taken for PCR analysis. ${ }^{12}$

The specific primer for blaOXA-51-like gene was (Forward - 5' TAA TGC TTT GAT CGG CCT TG; Reverse - 5'TGG ATT GCA CTT CAT CTT GG; Amplicon size 351 bp). The PCR assay was performed in a reaction mixture with total volume of
$25 \mu$ containing $15 \mu \mathrm{l}$ of master mix (TBG biotechnology Corp. USA), $0.15 \mu$ Taq polymerase (Solis BioDyne Germany), $1 \mu \mathrm{l}$ of forward and reverse primer each $(10 \mathrm{pmol} / \mu \mathrm{l}), 3 \mu \mathrm{l}$ of distilled water and $5 \mu \mathrm{l}$ of undiluted extracted DNA. The amplification condition was: initial denaturation at $95^{\circ}$ for 5 minutes, 30 cycles containing of denaturation at $95^{\circ}$ for 25 seconds, annealing at $60^{\circ}$ for 40 seconds, extension at $72^{\circ}$ for 50 seconds and final extension at $72^{\circ}$ for 6 minutes. The amplified products were subjected to electrophoresis in $1.5 \%$ agarose gel. $^{6}$ (Photograph-I)

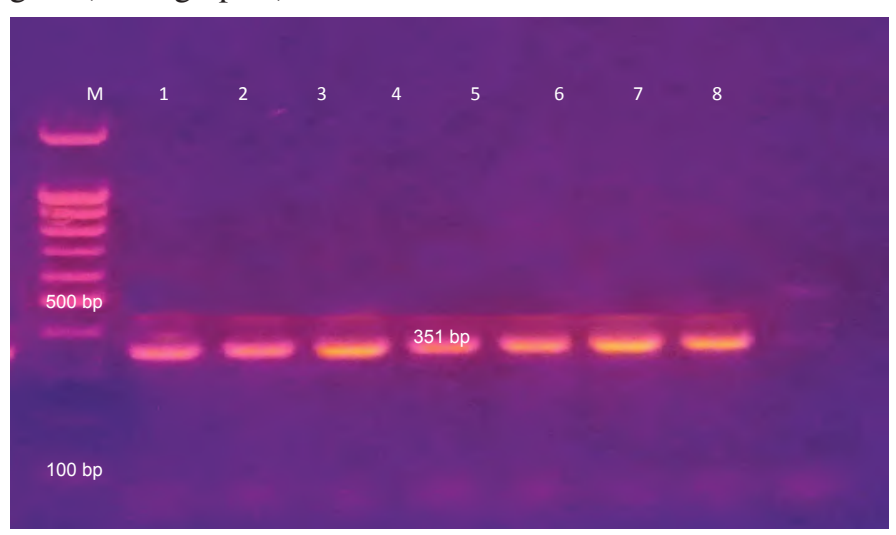

\section{Antimicrobial Susceptibility Testing}

A. baumannii were tested for antimicrobial susceptibility to ceftriaxone $(30 \mu \mathrm{g})$, cefotaxime $(30 \mu \mathrm{g})$, ceftazidime $(30 \mu \mathrm{g})$, cefepime $(30 \mu \mathrm{g})$, ciprofloxacin $(5 \mu \mathrm{g})$, cotrimoxazole $(1.25 / 23.75 \mu \mathrm{g})$, imipenem $(10 \mu \mathrm{g})$, meropenem $(10 \mu \mathrm{g})$, gentamicin $(10 \mu \mathrm{g})$, amikacin $(10 \mu \mathrm{g})$, netilmicin $(30 \mu \mathrm{g})$, piperacillintazobactum $(100 / 10 \mu \mathrm{g})$, ticarcillin-clavulanic acid $(100 / 10 \mu \mathrm{g})$, colistin (10 $\mu \mathrm{g})$, polymixin (300 units) (Oxoid, UK). The susceptibility was performed on Mueller Hinton Agar media (Merck, Germany) by modified disc diffusion method. ${ }^{13}$ Zone of inhibition were interpreted per recommendation of the Clinical Laboratory Standard Institute (CLSI) guidelines. ${ }^{14}$ In case of netilmicin zone of inhibition was used per BSAC standardized disc susceptibility testing method, ${ }^{15}$ for colistin and polymixin B zone of inhibitions were used per Gales et al (2001). ${ }^{16}$ E. coli (ATCC 25922) strains was used as the quality control reference strains according to CLSI.

\section{Study of Biofilm Formation}

Biofilm production of A. baumannii was done by tissue culture plate method (TCP) according to Toledo-Arana et al (2001). ${ }^{17}$

A. baumannii were grown overnight in Brain heart infusion broth (BHIB) (Becton Dickinson and company, USA) with $0.25 \%$ glucose at $37^{\circ} \mathrm{C}$. The broth culture was diluted at a ratio of $1: 40.200 \mu \mathrm{l}$ of this diluted culture suspension was inoculated in a sterile 96 well flat bottom polystyrene microtiter plate (Greiner Bio-One International, Kremsmunster, Austria). The positive control (Klebsiella pneunoniae ATCC 700603) and negative control (sterile BHIB- $0.25 \%$ glucose) were also added 
to microtiter plate in the same way. After overnight incubation at $37^{\circ} \mathrm{C}$, the wells were gently washed three times with $200 \mu \mathrm{l}$ of phosphate buffer saline (PBS). The plate was air dried, fixed with $200 \mu \mathrm{l} /$ well of $2 \%$ formalin at $4^{\circ} \mathrm{C}$ for 1 hour. After that, the wells were stained with $1 \%$ crystal violet for $15 \mathrm{~min}$. Afterward, the wells were rinsed under running tap water to remove the excess stain. Then $200 \mu \mathrm{l}$ ethanol-acetone (80:20, v/v) was added in each well to solubilize crystal violet. Each assay was performed in triplicate and repeated three times. The optical density (OD) at $630 \mathrm{~nm}$ was measured using ELISA plate reader (Plate reader, model-A4, serial no.-1910, Das, Italy).

The cut-off value (ODc) was calculated for each microtiter plate. ODc was of three standard deviations (SD) above the mean OD of the negative control: ODc $=$ average OD of negative control $+(3 \times \mathrm{SD}$ of negative control).

Final OD value of a tested strain was expressed as average OD value of the strain reduced by ODc value (OD = average OD of a strain - ODc). If a negative value is obtained, it should be presented as zero, while any positive value indicates biofilm production. ${ }^{18}$ Different steps of TCP method were depicted in photograph-II.

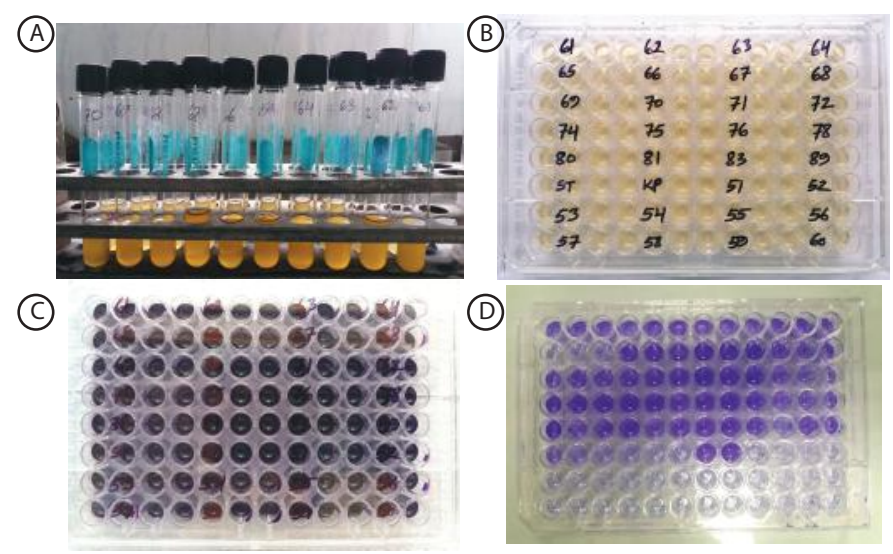

Data Analysis

The data were analyzed using SPSS software Version-23 (SPSS Inc., Chicago, IL, USA).

\section{Results}

\section{Isolates and Identification}

Total 108 Acinetobacter spp. were collected from which, 85 were identified as A. baumannii. Among 85 isolates, 39 (45.9\%) A. baumannii were obtained from tracheal aspirate, $13(15.3 \%)$ from wound swab, 5 (5.9\%) from pus, 18 (21.2\%) from blood, $9(10.6 \%)$ from urine, $1(1.1 \%)$ from pleural fluid. Majority of A. baumannii were isolated from tracheal aspirate (Table I).
Table-I: Distribution of $A$. baumannii Isolated from Different Type of Samples $(n=85)$

\begin{tabular}{lcc}
\hline Type of samples & $\begin{array}{c}\text { Number of A. baumanni } \\
\text { isolates }\end{array}$ & Percentage \\
\hline Tracheal aspirate & 39 & 45.9 \\
Wound swab & 13 & 15.3 \\
Pus & 05 & 5.9 \\
Blood & 18 & 21.2 \\
Urine & 09 & 10.6 \\
Pleural fluid & 01 & 1.2 \\
\hline Total & 85 & 100 \\
\hline
\end{tabular}

\section{Antimicrobial Susceptibility of $A$. baumannii}

Figure I showed antimicrobial resistance pattern of A. baumannii. A. baumannii showed $94.1 \%, 97.6 \%, 82.4 \%$, $87.1 \%, 63.5 \% 82.4 \% \quad 63.5 \%, 88.2 \%, 89.4 \%, 75.3 \%$, $85.9 \%, 80 \%, 82.4 \%, 88.2 \%$ resistance to ceftriaxone, cefotaxime, ceftazidime, cefepime, cotrimaxazole, ciprofloxacin, gentamicin, amikacin, netilmicin, imipenem, meropenem, pipercillin-tazobactum, ticarcillinclavulanic acid, colistin, polymixin B respectively.

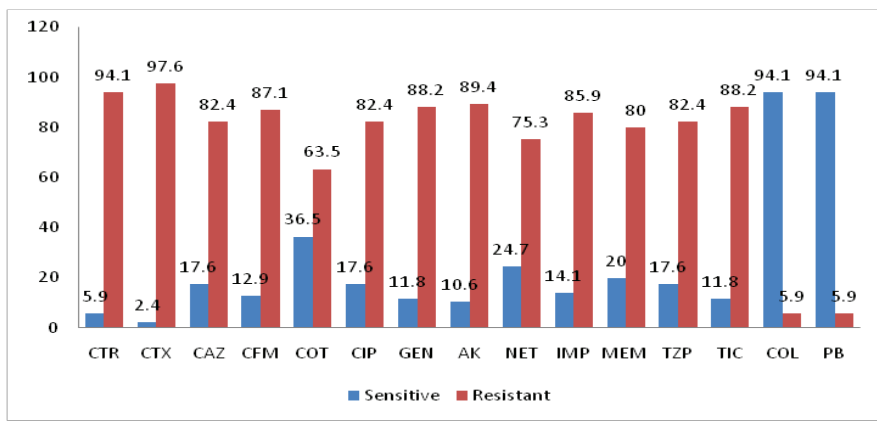

CTR: Ceftriaxon, CTX: Cefotaxime, CAZ: Ceftazidime, CFM: Cefepime, COT: Cotrimoxazole, CIP: Ciprofloxacin, GEN: Gentamicine, AK: Amikacin, NET: Netilmicin, IMP: Imipenem, MEM: Meropenem, TZP: Pipercillin-tazobactum, TIC: Ticarcillin-clavulanic acid, COL: Colistin, PB: Polymixin B

Figure-I: Antimicrobial Resistance Pattern of A. baumannii $(\mathbf{n}=\mathbf{8 5})$

Table II showed antimicrobial resistance pattern of A. baumannii in different type of samples. In tracheal aspirate, cefotaxime and ceftazidime were $100 \%$ resistant followed by ceftriaxone (97.4\%), cefepime (97.4\%), imipenem $(97.4 \%)$, meropenem $(97.4 \%)$, piperacillintazobactam (97.4\%), ticarcillin-clavulanic acid (97.4\%), amikacin $(97.4 \%)$, ciprofloxacin $(97.4 \%)$, gentamicin (94.7\%), netilmicin $(82.1 \%)$ whereas colistin and polymixin B showed only $2.6 \%$ resistance. In wound swab cefotaxime was $100 \%$ resistant followed by ceftazidime $(92.3 \%)$, ceftriaxone (92.3\%), cefepime (92.3\%), amikacin 
(92.3\%), ciprofloxacin (92.3\%), imipenem (84.6\%), meropenem $(84.6 \%)$, piperacillin-tazobactam $(76.9 \%)$, gentamicin (84.6\%). In blood cefotaxime was $88.9 \%$ resistant followed by ceftriaxone $(83.3 \%)$, gentamicin (77.8\%), amikacin (77.8\%), netilmicin (72.2\%). Pus, pleural fluid showed $100 \%$ resistance to all antimicrobial agent except colistin, polymixin $\mathrm{B}$.

Table-II: Antimicrobial Resistance Pattern of A. baumannii in Different Type of Samples $(n=85)$

\begin{tabular}{|l|c|c|c|c|c|c|}
\hline \multicolumn{1}{|c|}{ Antimicrobial agents } & \multicolumn{5}{|c|}{ Type of samples } \\
\cline { 2 - 7 } & $\mathrm{T} / \mathrm{A}(39)$ & $\mathrm{W} / \mathrm{S}(13)$ & Pus (05) & Blood (18) & Urine(09) & $\mathrm{PF}(01)$ \\
$\mathrm{n}(\%)$ & $\mathrm{n}(\%)$ & $\mathrm{n}(\%)$ & $\mathrm{n}(\%)$ & $\mathrm{n}(\%)$ & $\mathrm{n}(\%)$ \\
\hline Ceftriaxone (80)* & $38(97.4)$ & $12(92.3)$ & $5(100)$ & $15(83.3)$ & $9(100)$ & $1(100)$ \\
Cefotaxime (83)* & $39(100)$ & $13(100)$ & $5(100)$ & $16(88.9)$ & $9(100)$ & $1(100)$ \\
Ceftazidime (77)* & $39(100)$ & $12(92.3)$ & $5(100)$ & $12(66.7)$ & $8(89.9)$ & $1(100)$ \\
Cefepime (74)* & $38(97.4)$ & $12(92.3)$ & $5(100)$ & $10(55.6)$ & $8(89.9)$ & $1(100)$ \\
Cotrimoxazole (54)* & $30(76.9)$ & $9(69.2)$ & $5(100)$ & $7(38.9)$ & $4(44.4)$ & $1(100)$ \\
Ciprofloxacin (70)* & $38(97.4)$ & $12(92.3)$ & $5(100)$ & $9(50)$ & $5(55.6)$ & $1(100)$ \\
Gentamicin (75)* & $37(94.8)$ & $11(84.6)$ & $5(100)$ & $14(77.8)$ & $7(77.8)$ & $1(100)$ \\
Amikacin (76)* & $38(97.4)$ & $12(92.3)$ & $5(100)$ & $14(77.8)$ & $6(66.7)$ & $1(100)$ \\
Netilmicin (64)* & $32(82.1)$ & $8(61.5)$ & $5(100)$ & $13(72.2)$ & $5(55.6)$ & $1(100)$ \\
Imipenem (73)* & $38(97.4)$ & $11(84.6)$ & $5(100)$ & $12(66.7)$ & $6(66.7)$ & $1(100)$ \\
Meropenem (68)* & $38(97.4)$ & $11(84.6)$ & $5(100)$ & $7(38.9)$ & $6(66.7)$ & $1(100)$ \\
Piperacillin-tazobactum (70)* & $38(97.4)$ & $10(76.9)$ & $5(100)$ & $9(50)$ & $7(77.8)$ & $1(100)$ \\
Ticarcillin-clavulanic acid (75)* & $38(97.4)$ & $11(84.6)$ & $5(100)$ & $13(72.2)$ & $7(77.8)$ & $1(100)$ \\
Colistin (05)* & $1(2.6)$ & $0(0)$ & $0(0)$ & $4(22.2)$ & $0(0)$ & $0(0)$ \\
Polymixin B (05)* & $1(2.6)$ & $0(0)$ & $0(0)$ & $4(22.2)$ & $0(0)$ & $0(0)$ \\
\hline
\end{tabular}

Note: T/A: Tracheal aspirate; W/S: Wound swab; PF: Pleural fluid

* Indicate resistant

\section{Biofilm Formation}

Figure II showed that out of 85 A. baumannii, $78.8 \%$ produce biofilm and $21.2 \%$ were not produce biofilm.

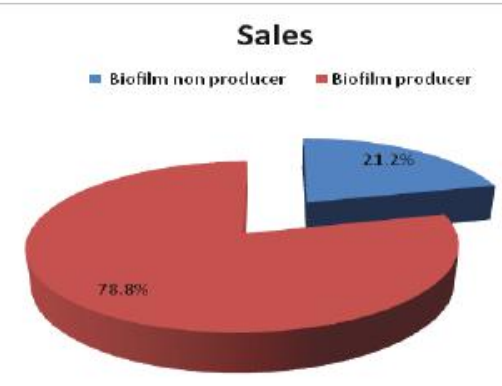

Figure-II: Biofilm production of $A$. baumannii
Table III showed Biofilm producer isolates of A. baumannii in different type of samples. A. baumannii showed $82.1 \%, 92.3 \%, 72.2 \%, 44.4 \%$ of biofilm formation in tracheal aspirate, wound swab, blood and urine respectively whereas showed $100 \%$ biofilm formation in Pus and pleural fluid.

Table-III: Biofilm producer isolates of $A$. baumannii in different type of samples

\begin{tabular}{lcc}
\hline Type of samples & $\begin{array}{c}\text { Biofilm producer isolates (67) } \\
\mathbf{n}(\%)\end{array}$ & $\begin{array}{c}\text { Non biofilm producer isolates (18) } \\
\mathbf{n}(\%)\end{array}$ \\
\hline Tracheal aspirate (39) & $32(82.1)$ & $07(17.9)$ \\
Wound swab (13) & $12(92.3)$ & $01(7.7 \%)$ \\
Pus (05) & $05(100)$ & $0(0)$ \\
Blood (18) & $13(72.2)$ & $05(27.8)$ \\
Urine (09) & $04(44.4)$ & $05(55.6)$ \\
Pleural fluid (01) & $01(100)$ & $0(0)$ \\
\hline Total (85) & $\mathbf{6 7}(\mathbf{7 8 . 8 \% )}$ & $\mathbf{1 8}(\mathbf{2 1 . 2 \% )}$ \\
\hline
\end{tabular}

\section{Discussion}

Most of the A. baumannii, 45.9\% of isolates were obtained from tracheal aspirates in this study. About $40.9 \%$ of A. baumannii in tracheal aspirates was reported by Barai et al (2010) ${ }^{19}$ in Ibrahim Medical College, Dhaka. Angoti et al (2016) ${ }^{20}$ reported $49.3 \%$ in Iran and Prabhu et al (2017) ${ }^{21}$ found $20.4 \%$ A. baumannii in tracheal aspirate in Nepal. In this study $15.3 \%$ of A. baumannii was isolated from wound swab whereas Angoti et al $(2016)^{20}$, Babapour et al (2016) ${ }^{22}$ isolated $11.6 \%$ and $10 \%$ of A. baumannii respectively. About $5.9 \%$ A. baumannii was isolated from pus in this study whereas 9.1\% was isolated by Prabhu et al (2017). ${ }^{21}$ In blood $21.2 \%$ of A. baumannii was isolated in this study and Angoti et al (2016) ${ }^{20}$ isolated $16.7 \%$ of A. baumannii. About $10.6 \%$ of $A$. baumannii was isolated from urine in this study whereas $6.5 \%$ and $17 \%$ was isolated by Angoti et al (2016) ${ }^{20}$ and Babapour et al (2016) ${ }^{22}$ respectively. In pleural fluid $1.1 \%$ of $A$. baumannii was isolated in this study and Babapour et al (2016) $)^{22}$ isolated 5\% of A. baumannii in pleural fluid.

In this study, the resistance of A. baumannii isolates was found as follows: ceftriaxone $(94.1 \%)$, cefotaxime (97.6\%), ceftazidime (82.4\%), cefepime $(87.1 \%)$, cotrimoxazole $(63.5 \%)$, ciprofloxacin $(82.4 \%)$, gentamicin $(88.2 \%)$, amikacin $(89.4 \%)$, netilmicin $(75.3 \%)$, imipenem $(85.9 \%)$, meropenem (80\%), pipercillin-tazobactum $(82.4 \%)$, ticarcillin-clavulanic acid $(88.2 \%)$. The resistance 
rate of colistin and polymixin B was $5.9 \%$. According to Prabhu et al (2017) $)^{21}$ A. baumannii was found resistance against ceftriaxone (93.2\%), cefotaxime (97.7\%), ceftazidime $(95.4 \%)$, cefepime (88.6\%), ciprofloxacin (97.7\%), cotrimoxazole (93.2\%), gentamicin $(52.3 \%)$, amikacin (43.2\%), and piperacillin/tazobactam (97.7\%). Angoti et al (2016) ${ }^{20}$ found A. baumannii was resistance against ceftriaxon $(99 \%)$, ceftazidime $(98 \%)$, cefepime (99\%), ciprofloxacin (99\%), cotrimoxazole (84\%), gentamicin $(77 \%)$, amikacin $(48 \%)$, imipenem $(99 \%)$, meropenem (99\%), colistin (11\%). According to Babapour et al (2016) $2294.87 \%$ of the A. baumannii were resistant to Ceftriaxon, $94.23 \%$ to cefepime, $89.10 \%$ to cotrimoxazole, $83.33 \%$ to gentamicin, $91.03 \%$ to imipenem, 95.51\% to Pipercillin-tazobactum, $93.59 \%$ to ticarcillinclavulanic acid. There was a high degree of susceptibility to PB (300).

In this study, 67 (78.8\%) of A. baumannii produce biofilm which was consistent with the results of Nahar et al $(2013)^{23}$ in Bangladesh, Gurung et al (2013) ${ }^{10}$ in India, Thummepak et al $(2016)^{24}$ in Thailand. Nahar et al $(2013)^{23}$ and Thummepak et al (2016) ${ }^{24}$ found $75 \%$ biofilm producing A. baumannii; Gurung et al (2013) $)^{10}$ found $73 \%$ biofilm producing A. baumannii.

In this study, $82.1 \%$ of $A$. baumannii produce biofilm in tracheal aspirate whereas Gurung et al (2013) ${ }^{10}$ and Rodríguez-Baño et al (2008) ${ }^{25}$ reported $51.2 \%$ and $32 \%$ of biofilm formation in respiratory samples where they include tracheal aspirate and sputum. In this study biofilm formation was detected only in tracheal aspirate. This may be the cause of highest rate of biofilm formation in tracheal aspirate in this study. In blood, $72 \%$ biofilm producing A. baumannii was detected and $100 \%$ was detected by Rodríguez-Baño et al (2008). ${ }^{25}$ About 44.4\% of biofilm producing A. baumannii was detected in urine in this study whereas Gurung et al (2013) ${ }^{10}$ and Rodríguez-Baño et al (2008) ${ }^{25}$ reported $25 \%$ and $69 \%$ of biofilm producing A. baumannii in urine. $100 \%$ of biofilm producing A. baumannii was detected in pleural fluid in this study. Gurung et al (2013) $)^{10}$ reported $100 \%$ biofilm producing A. Baumannii In sterile fluids (Pleural fluid and Peritoneal fluid).

\section{Conclusion}

This study demonstrated the ability of the clinical isolates of A. Baumannii to produce biofilm. Resistant to commonly used antibiotics such as cephalosporin, aminoglycosides, quinolone, carbapenem was also observed. Polymyxins were the only effective therapeutic agent in the study. This trend of multidrug resistance among A. Baumannii is a matter of concern.

\section{Ethical Approval}

Ethical clearance was taken from Institutional Review Board (IRB) of Bangabandhu Sheikh Mujib Medical University, Shahbag, Dhaka (No.BSMMU/2018/4348).

\section{Funding}

This work was supported by Department of Microbiology and Immunology, BSMMU and Research fund of Bangabandhu Sheikh Mujib Medical University (BSMMU).

\section{Acknowledgments}

The corresponding author is thankful to the Department of Microbiology and Immunology, Bangabandhu Sheikh Mujib Medical University (BSMMU) for providing necessary facilities to carry out the research.

\section{Disclosure}

The author reports no conflicts of interest in this work.

\section{Reference}

1. Dijkshoorn L, Nemec A, Seifert H. An increasing threat in hospitals: multidrug-resistant Acinetobacter baumannii. Nature reviews microbiology 2007; 5(12): 939-51.

2. Bergogne-Berezin E, Towner KJ. Acinetobacter spp. as nosocomial pathogens: microbiological, clinical, and epidemiological features. Clinical microbiology reviews 1996; 9(2): 148-65.

3. Turton JF, Shah J, Ozongwu C, et al. Incidence of Acinetobacter species other than A. Baumannii among clinical isolates of Acinetobacter: evidence for emerging species. Journal of clinical microbiology 2010; 48(4):1445-9.

4. Peleg AY, Seifert H, Paterson DL. Acinetobacter baumannii: emergence of a successful pathogen. Clinical microbiology reviews 2008; 21(3):538-82.

5. Al-Kadmy IM, Ali AN, Salman IM, et al. Molecular characterization of Acinetobacter baumannii isolated from Iraqi hospital environment. New microbes and new infections 2018; 21:51-7.

6. Woodford N, Ellington MJ, Coelho JM, et al. Multiplex PCR for genes encoding prevalent OXA carbapenemases in Acinetobacter spp. International journal of antimicrobial agents 2006; 27(4): 351-3.

7. Figueiredo S, Poirel L, Seifert H, Mugnier P, Benhamou D, Nordmann P. OXA-134, a naturally occurring carbapenem-hydrolyzing class $\mathrm{D} \beta$-lactamase from Acinetobacter lwoffii. Antimicrobial agents and chemotherapy 2010; 54(12): 5372-5. 
8. Gaddy JA, Actis LA. Regulation of Acinetobacter baumannii biofilm formation. Future Microbiology 2009; 4 (3): 273.8.

9. Opazo A, Dominguez M, Bello $\mathrm{H}$, et al. OXA-type carbapenemases in Acinetobacter baumannii in South America. The Journal of Infection in Developing Countries 2012; 6(04): 311-6.

10. Gurung J, Khyriem AB, Banik A, Lyngdoh WV, Choudhury B, Bhattacharyya P. Association of biofilm production with multidrug resistance among clinical isolates of Acinetobacter baumannii and Pseudomonas aeruginosa from intensive care unit. Indian Journal of Critical Care Medicine: Peer-reviewed 2013; 17(4): 214-8.

11. Tomaras AP, Dorsey CW, Edelmann RE, et al. Attachment to and biofilm formation on abiotic surfaces by Acinetobacter baumannii: involvement of a novel chaperone-usher pili assembly system. Microbiology 2003; 149(12):3473-84.

12. Dashti AA, Jadaon MM, Abdulsamad AM, et al. Heat treatment of bacteria: a simple method of DNA extraction for molecular techniques. Kuwait Medical Journal 2009; 41(2): 117-22.

13. Baurer AW, Kirby WH, Sherris JC, et al. Antibiotic susceptibility testing by standardizing single disc method. American Journal of Clinical Pathology 1996; 40:493-6.

14. Wayne PA. Clinical and Laboratory Standards Institute: Performance standards for antimicrobial susceptibility testing: 20th informational supplement. CLSI document M100-S20. 2018.

15. Andrews JM and BSAC Working Party on Susceptibility Testing FT. BSAC standardized disc susceptibility testing method. Journal of Antimicrobial Chemotherapy $2001 ;$ 48(suppl_1):43-57.

16. Gales AC, Reis AO, Jones RN. Contemporary assessment of antimicrobial susceptibility testing methods for polymyxin B and colistin: review of available interpretative criteria and quality control guidelines. Journal of clinical microbiology 2001; 39(1): 183-90.

17. Toledo-Arana A, Valle J, Solano C, Arrizubieta MJ, Cucarella C, Lamata M,. The enterococcal surface protein, Esp, is involved in Enterococcus faecalis biofilm formation. Applied and environmental microbiology 2001; 67(10): 4538-45.
18. Stepanovi S, Vukovi D, Hola V, et al. Quantification of biofilm in microtiter plates: overview of testing conditions and practical recommendations for assessment of biofilm production by staphylococci. Journal of Pathology, Microbiology and Immunology 2007; 115(8): 891-9.

19. Barai L, Fatema K, Haq JA, et al. Bacterial profile and their antimicrobial resistance pattern in an intensive care unit of a tertiary care hospital of Dhaka. Ibrahim Medical College Journal 2010; 4(2): 66-9.

20. Angoti G, Bandehpour M, Goudarzi H, Hajizadeh M, Zarringhalam Moghaddam M, Kouchaki A. Detection of Efflux Pump Genes (adeA, adeB, adeC and abeM) in Acinetobacter baumannii Isolated from Hospitalize Patients, North-west of Iran. Infection, Epidemiology and Microbiology 2016; 2(4): 8-11.

21. Joshi PR, Acharya M, Kakshapati T, Leungtongkam $\mathrm{U}$, Thummeepak R, Sitthisak S. Co-existence of bla OXA-23 and bla NDM-1 genes of Acinetobacter baumannii isolated from Nepal: antimicrobial resistance and clinical significance. Antimicrobial Resistance \& Infection Control 2017; 6(1): 1-7.

22. Babapour E, Haddadi A, Mirnejad R, et al. Biofilm formation in clinical isolates of nosocomial Acinetobacter baumannii and its relationship with multidrug resistance. Asian Pacific Journal of Tropical Biomedicine 2016; 6(6):528-33.

23. Nahar A, Anwar S, Saleh AA, et al. Virulence Factors and Antibiotic Susceptibility Pattern of Acinetobacter Species in a Tertiary Care Hospital in Bangladesh. Ibrahim Medical College Journal 2012; 6(1): 27-30.

24. Thummeepak R, Kongthai P, Leungtongkam U, et al. Distribution of virulence genes involved in biofilm formation in multi-drug resistant Acinetobacter baumannii clinical isolates. International Microbiology 2016; 19(2): 121-9.

25. Rodríguez-Baño J, Marti S, Soto S, et al. Biofilm formation in Acinetobacter baumannii: associated features and clinical implications. Clinical microbiology and infection 2008; 14(3): 276-8. 